\title{
FONÉTICA, LEXICOLOGÍA Y LEXICOGRAFÍA EN EL SIGLO XIX: TRES DISCIPLINAS INTEGRADAS EN LA OBRA DE CASTO VILAR (1894)
}

\author{
Ma. del Carmen Cazorla Vivas \\ UCM. Grupo de Investigación Lexicología y Lexicografía del español \\ mccazorl@filol.ucm.es
}

\begin{abstract}
Resumen
En la presente investigación nos aproximamos y presentamos una obra de finales del siglo XIX que abarca tres disciplinas distintas pero sumamente relacionadas, como son la fonética, la lexicologia y la lexicografía: Elementos de Fonética y Lexicología seguidos del vocabulario franco-español y españolfranco, Sevilla, Carlos de Torres y Daza, 1894. Esta integración de disciplinas en una misma obra (gramática y diccionario; fonética y diccionario; diálogos y gramática...) ha sido bastante habitual, sobre todo en los siglos XVHI y XIX, ya que, partiendo de objetivos fundamentalmente didácticos, ofrecían al usuario (principalmente escolares a partir de la segunda mitad del XIX) una herramienta de trabajo integral, para que sirviera de ayuda principalmente en sus traducciones y lecturas. Ahora describimos varios aspectos de esta obra (principalmente del apartado lexicográfico), que prácticamente no ha sido citada ni estudiada con anterioridad, lo que contribuirá a un mejor conocimiento de la hsitoriografía lingüistica.

PALABRAS CLAVE: historia de la lexicografía, fonética, lexicología, diccionarios español-francés, siglo XIX.
\end{abstract}

\section{Introducción}

Durante todo el siglo XIX el interés por el estudio de lenguas modernas irá en aumento y prueba de ello serán las numerosas obras que se publican dedicadas precisamente a la enseñanza y aprendizaje del español, francés, inglés, etc. Gramáticas, diccionarios, ortografías, diálogos... van a imprimirse y reimprimirse continuamente para aprovechar el amplísimo mercado que suponían los escolares. Es interesante señalar que en la presente investigación nos situamos a finales del siglo XIX, época en la enseñanza de segundas lenguas ya estaba instalada en los planes de estudio: recordemos que en 1836, el Duque de Rivas, en su Plan general de Instrucción pública, incluyó el estudio de lenguas vivas como materias obliga-
In this research we present a handbook published different phonetics, the lexicology and the lexicography: Elementos de Fonética y Lexicología seguidos del vocabulario franco-español y español-franco, Sevilla, Carlos de Torres y Daza, 1894. This integration of subjetcs at the same work (grammar and dictionary; phonetics and dictionary; dialogs and grammar ...) has been usual through centurys, especially in the XVIIth and XIXth century: with didactis objectives, they were offering to the user (principally student) a in hral handbook, in order to use as help principally several aspects of this work (principally of the mentioned not studied previously, with the aim to

KEYWORDS: history of the lexicography, phonetics, lexicology, dictionaries spanish-frecnh, 19th century. lexicographical section), that practically has not been contribute to a better knowledge of the linguistic historiography. 
torias en la instrucción secundaria, ya que hasta entonces se aprendían principalmente con maestros particulares. Con la Ley Moyano de 1857 se introduce en los Institutos de Segunda Enseñanza ${ }^{1}$.

En el ámbito del español y el francés, que es en el que nos vamos a centrar, aparecieron numerosos títulos a lo largo de los siglos XVIII y XIX, y es que debemos tener en cuenta que la lengua del país vecino, a partir del siglo XVIII había sido la lengua de cultura y era la primera lengua extranjera que se estudiaba en España. Pero no todas las obras publicadas corrían la misma suerte, ya que frente a algunas que tenían un éxito inmediato y se perpetuaban como manuales de referencia y de manejo continuo, otras pasaban casi directamente al olvido; o bien, muchas obras que en su momento sí se empleaban y tenían difusión no han merecido posteriormente el interés de los investigadores, o viceversa: obras con poco éxito que ahora se empiezan a rescatar para colocarlas en el lugar que les corresponde como eslabones necesarios de la historia de la lexicografia, de la gramática, de la fonética... En otros casos, es difícil conocer si realmente tuvieron o no éxito. Para el primer caso, baste simplemente con mencionar nombres como los de Oudin o Sobrino, autores cuyas gramáticas, diálogos y diccionarios conocieron un éxito completo y una gran repercusión a lo largo de décadas y décadas. En el segundo caso, el de obras con poca difusión, se enmarca el presente trabajo.

Nos vamos a detener en una publicación que no siguió la exitosa estela de los autores mencionados unas líneas más arriba; se trata de los Elementos de Fonética y Lexicología seguidos del Vocabulario franco-español y español-franco, de Casto Vilar y García, aparecida en Sevilla, Carlos de Torres y Daza, a finales del siglo XIX, concretamente en 1894.

En la portada leemos que el autor fue Catedrático Numerario de francés en el Instituto de Segunda Enseñanza de Sevilla. Sin duda fue el ejercicio de su profesión el principal motivo que lo impulsó a redactar una obra en la que integraba tres disciplinas diferentes, pero relacionadas. Así podía ofrecer a los usuarios (los escolares, principalmente) un manual variado y completo para acercarse a la lengua francesa y que sirviera de ayuda en las traducciones y lecturas; al mismo tiempo, facilitaba la tarea del profesor. De hecho, en su bibliografía observamos otros títulos que completaban, aún más, la enseñanza/aprendizaje del francés: un Método de la lengua francesa (dedicado al aspecto gramatical) (Sevilla, Impr. de Carlos de Torres y Daza, 1896); una Sintaxis francesa, seguida de un breve Tratado de Ortografia (Sevilla, Impr. de Carlos de Torres y Daza, 1895) o sus Trozos escogidos para la traducción del francés al castellano (Sevilla, Impr. de Díaz y Carballo, 1893) y del castellano al francés (Sevilla, Impr. de A. Pérez y Compañía, 1893). El argumento de, que todas estas publicaciones realmente constituían un todo para el aprendizaje de una lengua viene reafirmado, a nuestro juicio, por el hecho de que muchas de ellas aparecen incluso encuadernadas en un mismo volumen en muchas de las bibliotecas en las que se

1 En trabajos de investigadores como Cabezas y Herreras (1989: 17-25), Fernández Fraile y Suso López (1999: 81-82), Lépinette (2000: 177-178) o Cazorla Vivas (2005: 12-19) podemos profundizar en esta cuestión. El estudio de lenguas vulgares va a ir mereciendo mayor o menor atención en los planes de estudio según la situación política del momento, llegando incluso a desaparecer durante algunos años -antes de esta Ley figuraban las lenguas vivas, pero sólo como asignatura complementaria-. En todos los planes de estudio se va a destacar el fin práctico de las lenguas modernas. 
conservan ${ }^{2}$. La publicación conjunta de gramáticas y diccionarios, diálogos y gramáticas, diálogos y nomenclaturas... ha sido algo bastante habitual a lo largo de los siglos y con distintas lenguas ${ }^{3}$.

La obra que ahora presentamos aparece recogida en Palau, pero no en otros catálogos bibliográficos lingüísticos, ni ha sido citada ni estudiada en trabajos de investigación, que sepamos. Seguramente, el título, que comienza con "elementos de fonética, lexicología..." y en el que el apartado del vocabulario es uno más de los tres que ofrece, hizo que no se tuviera en cuenta a la hora de elaborar muchos de los repertorios lexicográficos (si bien tampoco tenemos notica de que haya sido estudiado por gramáticos, lexicólogos, etc.).

La obra de Vilar está dividida en tres partes. La estructura queda como sigue:

Título; Portada; Prólogo (5-7); Elementos de Fonética y Lexicología (9-62); Erratas (63); Título (Vocaculario francés-español y español-francés); Abreviaturas (I); VOCABULARIO FRANCÉS-ESPAÑOL (3-279); Título; VOCABULARIO ESPAÑOL-FRANCÉS (283-492)

El Prólogo que incluye Vilar (págs. 5-7) introduce las tres partes que integran la obra y justifica la presentación de las tres de manera conjunta. Comienza el preliminar dedicando unas líneas a la definición de 'gramática', que para él es 'la parte de la ciencia del lenguaje que trata de los sonidos de las palabras y de las frases o giros, ya en general [...], ya con aplicación a una lengua determinada' (pág. 5) ${ }^{4}$. Habla a continuación de las partes que integra esta disciplina: Fonética ('parte que se ocupa del sonido'), Analogía o Lexicología ('parte que se ocupa de las palabras aisladamente') y Sintaxis o Síntesis ('parte que se ocupa de las frases, o sea de las palabras en su conjunto'). El autor considera que la Fonética y la Lexicología son dos precedentes indispensables para el Vocabulario que va a ofrecer. Por un lado, la Fonética histórica da cuenta de las formas por las que una palabra ha pasado y permite compararla a otras formas ya conocidas. Por otro lado, el estudio de la Lexicología ahorra muchas consultas al diccionario:

La primera tarea del profesor en esta época en que las ramas del conocimiento humano se han multiplicado tan extraordinariamente, y en que hace falta saber tantas y tan útiles cosas, es simplificar en lo posible el trabajo del alumno, $y$, teniendo en cuenta el mucho tiempo que se pierde en el inútil e ímprobo trabajo de hojear lexicones y vocabularios, facilitarlo, ya que no pueda en absoluto suprimirse. (Pról., pág. 6).

Así, un estudio adecuado de las dos disciplinas mencionadas permitiría que se pudiesen eliminar del diccionario multitud de derivados y de palabras de otros idiomas que se parecie-

2 Por ejemplo, en el catálogo de la Biblioteca Pública del Estado/Biblioteca Provincial de Cádiz aparecen encuadernadas juntas la Sintaxis francesa con los Trozos escogidos del fracés al castellano (Signatura: XIX- 6135).

3 En muchas ocasiones los vocabularios que se incluían no eran alfabéticos, sino temáticos. Podemos citar, por ejemplo, para el inglés y el español y unos años antes de que apareciera la obra que nos ocupa, la Gramática española inglesa o método para aprender el inglés los españoles (1888) de Timoteo Cemborain, que incluye un tratado sobre la pronunciación inglesa; una gramática; unos principios teóricos gramaticales sobre las partes de la oración; una lista de verbos ingleses irregulares; una nomenclatura hispanoinglesa; unos fragmentos de obras escogidas y apartados dedicados a la redacción de cartas y correspondencia. Sobre esta obra puede verse García Aranda (2007) y sobre otras nomenclaturas Alvar Ezquerra (1987) o Ayala Castro (1998).

4 Discute así la opinión que Ferdinand Brunot vierte en su Précis de Grammaire... Historique de la Langue Française (1969) y de buena parte de la tradición gramatical anterior que la definían solo como 'arte de hablar y escribir correctamente'. 
ran a las correspondientes castellanas 5 . Pero no significa esto que vaya a suprimir todos los derivados o todas las voces que presenten alguna semejanza con las españolas: en el momento en que aparece cualquier duda sobre su significado, se decide por la inclusión del vocablo.

Respecto a los elementos multiverbales, argumenta que los más usuales se incluyen en el apéndice de otra de sus obras, Método de la Lengua Francesa, por lo que remite a su consulta cuando sea necesario. Por tanto, serán muy escasos en su vocabulario (aunque alguno hay, como veremos más adelante).

En el apartado de las voces técnicas, indica que sólo incluye las más usuales, porque son $\tan$ abundantes, sobre todo en determinadas ciencias, que se podría formar un vocabulario amplísimo sólo con ellas. A pesar de esta intención, lo cierto es que aparecen bastantes, como también veremos, en proporción con la nomenclatura limitada que ofrece.

\section{Elementos de fonética ${ }^{6}$}

Vilar, antes de adentrarse en el análisis de las reglas fonéticas que ha seguido el francés desde su evolución del latín, y con alusiones al español, ofrece una introducción en la que comienza hablando de las transformaciones del latín, que derivaron en diferentes lenguas. Dichas transformaciones no fueron igualmente profundas, ya que, por ejemplo, los cambios fueron en Francia mucho más radicales que en España.

Para Vilar, las variaciones son, principalmente, de dos clases: una de ellas es la espontánea, irreflexiva, la que lleva a cabo el pueblo que habla un idioma, obedeciendo a leyes y tendencias de que él mismo no se da cuenta; la segunda es la que realizan los eruditos, cuidadosos de enriquecer el lenguaje y depurarlo, obedeciendo a leyes y tendencias distintas de las vulgares.

Como consecuencia de estas dos tendencias nacen los llamados "dobletes", es decir, la doble transformación de una misma palabra latina (p. e. del lat. auscultare surge la voz vulgar o popular, escuchar y la científica o erudita auscultar).

En español sería difícil fijar a cuál de estos dos grupos pertenecen muchas palabras, pero en francés la diferencia es fácilmente perceptible, puesto que las palabras de formación popular conservan el acento en la misma sílaba que las palabras latinas que representan, mientras que las de formación erudita no lo conservan generalmente (porticum $>$ porche [popular], portique [erudita]).

Otra de las particularidades es que en estas voces, la sílaba o sílabas que seguían a la acentuada, o han desaparecido totalmente, o han sido reemplazadas por otras teniendo por vocal una e muda. Esto explica por qué toda dicción en francés es aguda, a no ser que consideremos como llana o grave la terminada en $e$ muda. En cualquier caso, las esdrújulas no existen en la lengua francesa.

5 Así, por ejemplo, indica que el estudioso, gracias a sus conocimientos de fonética, sabe que la $l$ entre vocal y consonante se ha vocalizado en $u$, y que la $e$ representa una $a$ las más veces, y por tanto no necesita buscar en el diccionario la palabra aube, que para él representa otra voz: alba, idéntica a la castellana y procedente de la latina albam. Critica, asimismo, la pérdida que supone, en muchos diccionarios plurilingües, la inclusión de voces sumamente parecidas en los idiomas tratados, como por ejemplo, blanc, soldat o abdiquer.

6 No tenemos noticia de que aparecieran, en fechas próximas a la obra que nos ocupa, muchas publicaciones dedicadas a la fonética o la fonología. En el manual de Quilis apenas hemos localizado dos títulos: Fernando Araujo, Estudios de fonética castellana, Toledo, 1894 y F. Hanssen, Elementos de fonología castellana, Cultura Universitaria, Caracas, 106, 1900. 
El autor expone que en su análisis se va a centrar en el caudal de voces popular, ya que ofrece más interés al filólogo: el uso culto presenta pocos inconvenientes en su formación y obedece a escasas reglas.

Expone a continuación, pormenorizadamente, las reglas que siguieron las vocales y consonantes francesas en su evolución?.

\section{Elementos de lexicología}

$\mathrm{Al}$ introducir sus elementos de lexicología, Vilar se esfuerza en señalar la conexión que existe entre las disciplinas por él tratadas en este libro, y así, estima que si la Fonética considera a la palabra como un conjunto de sonidos y la Lexicología la considera como representante de ideas, ambas parcelas no deben separarse si, como es fácilmente demostrable, cada grupo fonético o entidad silábica corresponde a una idea. Así que, en cada palabra hay un sonido o grupo de sonidos que encierra la idea principal, y a este sonido le preceden o siguen otros sonidos o sílabas que lo enriquecen. Vilar arguye el ejemplo de la voz inconstitucionalísimamente, y va separando todos sus componentes (prefijos y terminaciones: in-, -mente, -isima, -al, -cion, con-) hasta quedarse con stitu, del verbo latino statuo, is, donde como en otros muchos verbos (ya sea para indicar la frecuencia, ya el tiempo pasado) se duplica alguna letra de la raíz o radical. El último análisis nos deja el grupo sti, que indica fijeza, permanencia, existencia y otras ideas análogas, que se encuentran en otras lenguas de origen ario (alemán, griego).

El conocimiento de todos los elementos accesorios que anteceden al núcleo o raíz (prefijos, sufijos) deben preceder al estudio del léxico, ya que su conocimiento ahorraría en gran manera la consulta de diccionarios. Por esta razón incluye este apartado, en el que enumera y describe los prefijos y sufijos, que efectivamente precede al vocabulario franco-español. Los accidentes de género, número, persona, etc. pertenecen, sin embargo, a la gramática y es ahí donde los ha estudiado.

\section{Vocabulario español-francés y francés-español ${ }^{9}$}

El número de entradas aproximado es de 15.500 voces en la dirección español-francés y unas 17000 en la parte francés-español. Es un número global bastante inferior a otros diccionarios de la centuria ${ }^{10}$.

En la macroestructura, y a pesar que la cantidad limitada de voces que incluye, y de tratarse de un diccionario general, llama la atención la aparición bastante frecuente de vo-

7 Muchos diccionarios que aparecían como obras independientes, sin estar incuidos en otras obras de conjunto ni acompañar, por tanto, a otros apartados fonéticos o gramaticales, sí incluian a menudo la pronunciación figurada en el interior de los artículos, lexicográficos. Pueden verse, a este respecto, trabajos como los de Bruña Cuevas (1996, 2002 y 2005). En el caso de las Nomenclaturas, es decir, los vocabularios temáticos, no solían ofrecer la pronunciación, pero hay algunos casos en que sí. Véase, por ejemplo, la investigación de García Aranda (2005-2006).

8 Por ahora no indagamos más en este ni en el anterior apartado ya que el objeto último de estudio del presente trabajo es, principalmente, el apartado lexicográfico.

9 Para realizar esta investigación hemos trabajado con la totalidad de la letra $\mathrm{A}$ en las dos direcciones, con lo que resulta para la parte español-francés una cantidad de unos 2.170 artículos y para la parte francés-español de unos 1.750 . 10 Muy lejos de las cantidades que ofrecen grandes obras hispano-francesas, como las de Domínguez (unas 89.000 en la dirección francés-español) o Fonseca (unas 38.000); e incluso de los repertorios denominados "de bolsillo" o portátiles contenían, en prácticamente todos los casos, más de 20.000 (Cazorla Vivas: 2005 y 2006). 
ces pertenecientes a realidades lejanas y exóticas y que, forzosamente, debían tener un uso bastante limitado (a no ser en lecturas), cuya inclusión no se entiende demasiado bien en un repertorio limitado como éste, pero que, desde luego, sí se enmarca en la tradición decimonónica de los grandes diccionarios, tanto monolingües como bilingües, que circulaban ${ }^{11}$ y que siguiendo la línea enciplopédica francesa daban cuenta de multitud de voces poco generales. Nos referimos a artículos como los siguientes:

(francés-español) $^{12}$

abacus, m. Bastón usado entre los Templarios. abdélavi, m. Melón de Egipto.

achit, m. Achit, viña de Madagascar. aga, m. Agá, funcionario turco. || Bot. Cardo de Siria.

alemdar, $m$. Alendar, abanderado turco.

alépine, f. Agalla de Alepo.

alueromancie, f. Aleuromaucia, adivinación por medio de la harina.

alfa, m. Alfa, gran sacerdote senegalés. \|| Esparto. alibanies, pl. Alibanias, telas indias. allemande, f. Alemanda, dana iriginaria de Alemania.

alogien, $\mathrm{m}$. Alogo, hereje que negaba la eternidad del Verbo.

alphanet, m. Halcón de Túnez.

areca, $f$. Arec, fruta india.

aspre, $m$. Aspro o áspero, moneda de Turquía. assaky, f. Asaky, primera sultana.

aulique, adj. Áulico: se dice del consejo y consejeros de Alemania.

ayac-divani, Hist. Ayac-diván, junta turca.

En la dirección español-francés apenas localizamos voces de este tipo; el léxico escogido por Vilar para esta segunda parte es mucho más común y cercano; apenas podemos señalar el artículo: 'añafil, f. Trompette des Maures'.

En la microestructura, en la dirección español-francés gran parte de los artículos contienen únicamente uno o varios equivalentes sinonímicos, si bien hallamos definiciones más extensas o artículos que incluyen varias acepciones. En la dirección francés-español aumenta considerablemente el número de artículos más amplios. De manera más concreta, podemos establecer los siguientes esquemas definicionales:

1) ENTRADA + CATEg. GRAMATICAL + EQUIVALENTE(s):

(español-francés)

abaceria, f. épicerie.

abada, f. Rhinocèros.

abanico, $\mathrm{m}$. Eventail.

abeja, f. Abeille.

(francés-español)

Abas, m. Aguacero.

Abbé, m. Abad. \| Clérigo. \|| Abate.

Abricot, m. Albaricoque. abellacar v. Mépriser.

acontecer, v. Arriver, survenir. achicado, adj. Enfantin, pueril. alboroto, m. Tumulte, émeute.

Accointer ( $s$ '), r. Compadrar, hacerse compinche. Aigrette, f. Cresta. Penacho. || Piocha.

Août, m. Agosto. || Siega.

Apetissement, m. Apocamiento.

11 Puede verse el trabajo sobre diccionarios del siglo XIX de Azorín (1996). Un claro ejemplo de este enciplopedismo está presente en las obras, tanto monolingüe como bilingüe, de R. J. Domínguez. Sobre este autor, pueden verse, entre otros, los trabajos de Esparza (1999), Seco (2003) o Quilis (2004 y 2007) para su diccionario monolingüe, y Bruña Cuevas (2003), Cazorla Vivas (2003) o Quilis (2007) sobre su repertorio bilingüe.

12 Ninguna de las siguientes voces aparece luego en la dirección español-francés. 
2) entrada + Categ. Gramat. + estructura defintorla: (esp.-fr.) “(celui) Qut ..." / (fr.-esp.) (el/La/lo) que:

(español-francés)

abigotado, adj. Qui porte des moustaches. abizcochado, adj. Qui a du biscuit.

ablandador, $\mathrm{m}$. Qui amollit.

abrasador s.m. Qui embrasse.

(francés-español)

abonnataire, $\mathrm{m}$. El que logra una concesión por vía de abono.

affaiteur, m. El que cría aves de rapiña.

3) ENTRADA + CATEg. GRAMAT. + DEFInición EXTENSA:

(español-francés)

abarca, f. Chaussure rustique; espèce de sandale.

abarquillado, adj. Roulé en forme d'oublie. abejón, m. Mâle de la reine des abeilles. abolsado adj. Plissé comme une bourse. abonaré, $\mathrm{m}$. Bon payable pour une somme. acaudillar, v. Commander des gens de guerre. accidentado, adj. Malade par suite d'un syncope. acequiado, adj. Lieu arrosé par des canaux. abreviaduria, f. Fonctions du rédacteur de brefs. acordada (carta), f. Lettre par laquelle un tribunal supérieur reprend quelqu'un ou demande à s'informer de quelque chose.

adarve, $m$. Espace sue les anciens murs derière les créneaux.

aguaderas, f. pl. Paniers pour cruches pleines d'eau dont on charge les bêtes de somme.

(francés-español)

abéchement, abecquement o abéquement, $\mathrm{m}$. La acción de meter a un pájaro la comida en el pico.

agréé, $\mathrm{m}$. El abogado que puede informar en los tribunales de comercio.

ahah, m. Foso profundo al extremo de una alameda.

aiguiserie, f. Taller donde se afilan las armas blancas.

allésoir, $\mathrm{m}$. Instrumento para ensanchar el calibre de un cañón. alberquero, $\mathrm{m}$. Qui prend soin des routoirs et des réservoirs d'eau.

alobunado, adj. Qui ressemble au loup.

amelonado, adj. Qui a la forme du melon.

araigneux, euse, adj. Lo que se parece a la telaraña.

apailleur, $\mathrm{m}$. El que busca oro en los ríos.

apartadero, $\mathrm{m}$. Lieu où l'on peut se retirer pour laisser passer quelqu'un.

apedreadero, $\mathrm{m}$. Lieu où les enfants jouent ou se battent à coups de pierres.

arandanedo, $\mathrm{m}$. Terrain sombre et humide planté d'areilles.

arrancadero, m. Endroit d'où l'on s'élance à la course.

arrepisto, $\mathrm{m}$. Secoud des chiffons pour fabriquer du papier.

asonantar, v. Composer des vers avec la même assonance.

aspado, $\mathrm{m}$. Homme qui ne peut se mouvoir dans ses vêtements.

atasajar, v. Couper de la chair en morceaux pour la boucaner.

ataurique, m. Ornement en plâtre dans la frise des bâtimes maures en Espagne.

anamorphose, f. Pintura que vista de diferentes puntos representa objetos diversos.

aphronatron, $\mathrm{m}$. Carbonato de sosa en ciertas paredes húmedas.

archée, f. Archeo, principio vital que, suponen algunos, existe en cada órgano humano.

aymara, $\mathrm{m}$. Aimara, idioma que hablaban los aimaras del Perú. 
4) ENTRADA + CATEG. GRAMAT. + EQUIVALENTE + DEFINICIÓN EXTENSA:

(francés-español)

abacot, m. Ábaco, adorno de cabeza que usaban los reyes de Inglaterra.

abée, f. Bocal, canal que conduce las aguas de un molino.

abluer, a. Lavar, avivar la escritura por medio de $\mathrm{n}$ líquido.

affinoir, m. Peine, rastrillo con que se limpia el cáñamo. agasillis, m. Agasilis, arbusto que da la goma moniaca.

aggrave, m. Agravación, segunda amonestación de una censuraa eclesiástica. $\|[\ldots]$

aleuromancie, f. Aleurmaucia, adivinación por meido de la harina.

alogien, m. Alogo, hereje que negaba la eternidad del Verbo.

5) ENTRADA + CATG GRAMAT. + $1^{\text {a }}$ ACEPCIÓN $\| 2^{\mathrm{a}}$ ACEPCIÓN $\|$ [3 ${ }^{\mathrm{a}}$ Y SIGUIENTES ACEPCIONES]:

(español-francés)

abadía, f. Abbaye || Presbytère.

abalanzar, v. Pousser || Lancer.

abertura, f. Ouvertur \| Fente.

abonar, v. Accréditer || Cautionner || Engraisser

(agr.)

abono, m. Garantic. || Abonnement. || Engrais

(agr.) $\mathrm{m}$.

altibajo, m. Terrain inégal. \| Coup d'épée de haut en bas. || Vicissitude. aparejar, v. Préparer. || Enharnacher. \|| Apprêter. II Appareiller.

apocar, v. Diminuer. || Fig. Réduire, resteindre. || Abattre l'esprit. || S'humilier.

arranque, m. Déracinement. || Arrachement. || Mouvement impétueux.

asistenta, f. Demoiselle des dames d'honneur à la cour. || Servante des religieuses militaires. aventajado, m. Appointé. \| Adj. Superbe.

avistar, v. Apercevoir. \|| Avistarse: S'aboucher.

(francés-español)

abreuvage, m. Regadío. \| Riego. \| Acción de abrevar.

aiguille, f. Aguja. || Obelisco. \|| Campanario alto y muy puntiagudo. || Mano del reloj. || Fiel de las balanzas. || Brujula.

ajourner, a. Aplazar. | Citar. || Suspender.

alerte, f. Alarma, rebato. \| Prevención. \| adj. Vigilante. || Avispado. || adv. Cuidadosamente. || interj. ¡Alerta! ¡cuidado!

allier, $\mathrm{m}$. Red para codornices $\|$ a. Ligar, mezclar. || Unir, juntar. || r. Emparentar. || Aliarse.

appointé, e, adj. Contrapuesto. \|| Asalariado. \| Señalado. || Aventajado.

appuyer, a. Sostener. || Apoyar. || Proteger. || cargar. || Insistir.

No encontramos apenas elementos multiverbales (expresiones, ejemplos o refranes); el propio autor indica en el prólogo que éstos aparecen en un apéndice de su otra publicación, la ya citada Método de la lengua francesa. Sí se incluyen algunas variantes léxicas o expresiones lexicalizadas como: agalla de ciprés, en agraz, hacer añicos, ayuda de cámara, ayudante de campo, todas ellas en la dirección español-francés; o s'en faire accroire, aide de camp, aisdélié, ais de boutique, mettre quelqu'un aux ambles, pont aux ânes, faire le pot à deux anses, faire danser l'anse du panier, il pleut à verse, à l'aveugle, en aveugle.

Podemos destacar la aparición, de manera bastante habitual, del entorno contextual, del ámbito léxico al que pertenece la voz en cuestión. Generalmente esta información aparece 
entre paréntesis, si bien su ocurrencia se limita prácticamente a la dirección español-francés, ya que en la parte francés-español las indicaciones vienen insertas en la definición, sin separación mediante paréntesis, como por ejemplo: 'ayra, m. Zool. Aira, zorro' o 'ayri, m. Bot. Airi, palmera' o 'able, m. Albur o breca, pez de río.'.

Algunos de estos artículos en la dirección español-francés son:

(español-francés)

abocado, adj. Qui a de bouquet (en parlant du vin).

abridero $\mathrm{m}$. Pavie (pêche).

acanillado, adj. Vergé (le papier).

alano, m. Dogue (chien).

alfil, m. Fou (du jeu d'échecs).

altamisa, $\mathrm{f}$. Armoise (herbe).

alzadamente, adv. Un peu élévé (du prix). $\|$ En

gros.

amargoso, adj. Amer (un peu). apabilar, v. Préparer Ila mèche d'un cierge, etc. \| apabilarse; S'obscurcir (en parlant de la flamme d'une chandelle).

apacentadero, m. Pâturage (lieu); pâtis, pacage. apitonar, v. Piquer, percer. \| Commencer à poindre (en parlant des cornes). || Bourgonner. ariete, $\mathrm{m}$. Bélier (machine).

arroyar, V. Entraîner la terre (en parlant d'une ravîne). Se nieller les plantes céréales. austro, m. Auster (vent).

En el apartado de las marcas lexicográficas constatamos una enorme diferencia entre las dos direcciones de la obra: en la letra A de la parte español-francés, contamos 36 indicaciones de especialidad, más una diafásica (de estilo familiar, concretamente). Sin embargo, en la dirección francés-español, y sin duda porque la obra iba dirigida a españoles que deseaban aprender o mejorar su francés, contamos un amplísimo número de artículos marcados: hay hasta 280 marcaciones diatécnicas, de hasta 49 ámbitos diferentes, más otras indicaciones diadrónicas (10) y diafásicas (32). Todas vienen señaladas a continuación del equivalente correspondiente, en abreviatura y entre paréntesis. Veamos el cuadro-resumen resultante y a continuación, a modo de muestra, algunos de estos artículos marcados en las dos direcciones:

(español-francés)

\begin{tabular}{|l|l|l|l|l|}
\hline & \multicolumn{2}{|c|}{$\begin{array}{c}\text { Marcas de } \\
\text { especialidad }\end{array}$} & \multicolumn{2}{c|}{$\begin{array}{c}\text { Marcas } \\
\text { diafásicas }\end{array}$} \\
\hline & Agr. & 3 & & \\
Bot. & 11 & & \\
& Mar. & 12 & Fam. & 1 \\
& Mit. & 1 & & \\
& Mús. & 2 & & \\
& Zool. & 6 & & \\
\hline
\end{tabular}


De especialidad:

abarloar, v. Amarrer (mar.)

abedul, m. Bouleau (bot.)

abonar, v. Accréditer. || Cautionner. || Engraisser (agr.)

abono, m. Garantie. || Abonnement. || Engrais (agr.) $\mathrm{m}$.

acebo, m. Houx (bot.)

Diafásicas:

avergonzar, v. Faire honte, faire rougir. || Fam. Équinauder.

(francés-español)

\begin{tabular}{|c|c|c|c|c|c|c|c|c|c|c|c|c|}
\hline & Marcas & e es! & cialidad & & & & & & $\begin{array}{l}\text { Marc } \\
\text { diacrc }\end{array}$ & & $\begin{array}{l}\text { Marca } \\
\text { difásio }\end{array}$ & \\
\hline $\begin{array}{l}\text { Letra } \\
\text { A }\end{array}$ & $\begin{array}{l}\text { Agr. } \\
\text { Alb. } \\
\text { Anat. } \\
\text { Arq. } \\
\text { Art. } \\
\text { Artill. } \\
\text { Astr. } \\
\text { Blas. } \\
\text { Bot. } \\
\text { Carp. } \\
\text { Cerraj. } \\
\text { Cir. } \\
\text { Coc. }\end{array}$ & $\begin{array}{l}14 \\
3 \\
4 \\
26 \\
8 \\
3 \\
4 \\
8 \\
30 \\
1 \\
1 \\
5 \\
1\end{array}$ & $\begin{array}{l}\text { Com. } \\
\text { Encuad. } \\
\text { Equit. } \\
\text { Farm. } \\
\text { Feud. } \\
\text { Fil. } \\
\text { Fís. } \\
\text { Fisiol. } \\
\text { For. } \\
\text { Fort. } \\
\text { Geom. } \\
\text { Gram. } \\
\text { Hidr. }\end{array}$ & $\begin{array}{l}10 \\
1 \\
3 \\
1 \\
3 \\
1 \\
2 \\
1 \\
16 \\
2 \\
2 \\
1 \\
1\end{array}$ & $\begin{array}{l}\text { Hist. } \\
\text { H i s } t \\
\text { nat. } \\
\text { Impr. } \\
\text { Jard. } \\
\text { Jurisp. } \\
\text { Mar. } \\
\text { Med. } \\
\text { Mil. } \\
\text { Min. } \\
\text { Mist. } \\
\text { Mit. } \\
\text { Mont. }\end{array}$ & $\begin{array}{l}1 \\
6 \\
3 \\
2 \\
7 \\
63 \\
8 \\
7 \\
3 \\
1 \\
2 \\
1\end{array}$ & $\begin{array}{l}\text { Mús. } \\
\text { Pesc. } \\
\text { Poes. } \\
\text { Poét. } \\
\text { Quím. } \\
\text { Relig. } \\
\text { Sast. } \\
\text { Teol. } \\
\text { Vet. } \\
\text { Zap. } \\
\text { Zool. }\end{array}$ & $\begin{array}{l}7 \\
2 \\
1 \\
1 \\
1 \\
1 \\
1 \\
3 \\
4 \\
1 \\
2\end{array}$ & Ant. & 10 & $\begin{array}{l}\text { Fatn. } \\
\text { Joc. } \\
\text { Vulg. }\end{array}$ & $\begin{array}{l}24 \\
2\end{array}$ \\
\hline
\end{tabular}

De especialidad:

abrivent, $m$. Abrigaño. // Jard. Cobertizo de pja. about, m. Carp. Cabeza, extremo de un madero. abréyer, a. Mar. Abrigar, quitar el viento, formar socaire.

accense, f. Jurisp. Censo.

accoudoir, m. Reclinatorio = Arq. Antepecho de una ventana.

accul, m. Rincón. \| Artill. Estaca que se clavaba en tierre para impedir el retroceso del cañón. achéron, m. Mit. Aqueronte. || Poét. Infierno, muerte. acyrologie, f. Gram. Impropiedad.

aquilinées, f. pl. Hist. nat. Aquilinias o falconias. ariès, m. Astr. Aries.

avant-corps, m. Arq. Arimez. \| Cerraj. Antecuerpo

Diacrónicas:

acensement, $\mathrm{m}$. ant. Arrendamiento a censo.

adoubert, n. ant. Componer.

Diafásicas:

ablativo, adv. Fam. Todo junto, confusamente. accordailles, f. pl, vulg. Esponsales. acordar, v. Arrêter (mus). \| Convenir. || Résoudre. corse: Se rappeler.

jour.

avión, m. Martinet (zool.) 
Resulta, evidentemente, un número bastante alto de términos marcados, sobre todo teniendo en cuenta el vocabulario limitado que recoge esta obra en comparación con otras de la época (como hemos comentado más arriba).

Pero a pesar del evidente interés que ofrece la abundancia de léxico técnico incluido, nos llama la atención cómo en una misma familia de voces, algunas de ellas llevan algún tipo de marcación, mientras que otras no la incluyen, cuando, a nuestro juicio, tendrían el mismo valor técnico. Ocurre, por ejemplo, con artículos como los siguientes:

(español-francés)

arrayán, m. Myrthe (bot.)

arrayonal, m. Lieu planté de myrthes.

(francés-español)

accorder, a. [...] || Mmús. Acordar, templar los instrumentos.

accordeur, m. Afinador.

accordoir, m. Templador.

adjurateur, $\mathrm{m}$. Conjurador, exorcista.

adjuration, f. Teol. Conjuro, exorcismo.

adjurer, a. Conjurar.

alène, f. Art. Lesna.

alénier, m. Lesnero.

alunation, f. Alunación, formación de alumbre. aluner, a. Art. Alumbrar dar un baño de agua de alumbre.

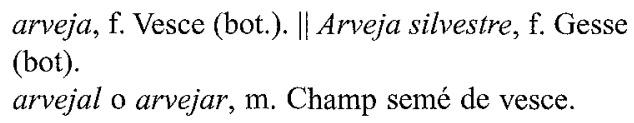

ancrage, m. Mar. Anclaje. \| Ancladero, fondeadero.

ancre, f. Mar. Ancla. I| met. Áncora.

ancrer, a. Anclar, echar el ancla.

arage, $\mathrm{m}$. agr. Aradura.

araires, m. pl. Instrumentos de agricultura. II

Arado.

arc-bouter, a. Apuntalar.

arc-doubleau, m. Arco apuntado.

arceau, m. Arq. Arco de bóveda, ventana o puerta.

arrière-point, m. Sast. Pespunte, punto atrás. arrière-pointeuse, f. Pespuntadora.

O bien hallamos voces que por su uso y definición deberían aparecer marcadas y no es así, como ocurre con acordada o agréé (pueden verse más arriba) en las que no viene ninguna marca de 'Jurisp.', en ataurique (vése más arriba) no aparece la marca de 'Art.', o en 'avives, f. pl. Adivas, inflamación de las glándulas de los caballos' no aparece marca de 'Veter.', mientras que sí viene en otras voces similares.

\section{Conclusiones}

La obra que nos ocupa reviste interés para la historiografía lingüística porque sigue claramente la línea didáctica que desde siglos antes procuraba ofrecer a los usuarios herramientas, lo más completas y útiles posible para el aprendizaje de una segunda lengua. Vilar, que era profesor de instituto, conocía las dificultades y necesidades que tenían los alumnos y así ofrece un manual que les sirve de ayuda en sus composiciones y traducciones; de ahí, seguramente, la inclusión de cierto léxico poco general y la abundancia de tecnicismos que hallamos entre sus páginas, aspecto este último, de los más interesantes que encierra.

La nomenclatura elegida es limitada, frente a lo habitual en otras obras lexicográficas, pero en este caso hay que tener presente que el vocabulario no aparece de manera independiente, sino formando parte de un conjunto más amplio. 
Respecto a las posibles fuentes que guiaran a Vilar en la composición de su vocabulario, en nuestras investigaciones no hemos encontrado ninguna obra que sea lo suficientemente parecida como para hablar de modelo directo, así que tendremos que pensar, mientras que no surjan nuevos datos, en la propia competencia del autor para redactar su obra. Dicho esto, prácticamente ningún repertorio lexicográfico ha surgido de la nada, por lo que sí podemos hablar de otro vocabulario que le pudo servir de base o ayuda: se trata del diccionario, también poco conocido, de Soler Arqués ${ }^{13}$, obra parecida a la de Vilar, ya que muchos artículos son iguales en los dos, y otros son muy similares, pero generalmente más breves en Vilar. La nomenclatura no es exactamente igual, ya que hay añadidos y supresiones por ambas partes (si bien la macroestructura en Vilar en menos numerosa). En cualquier caso, y aunque las similitudes son razonables, tampoco tenemos constancia de que el diccionario de Soler Arqués llegara a manos de Vilar, sobre todo porque apareció solo un año antes y tampoco tuvo una gran difusión. Las restantes obras con las que hemos realizado el cotejo (gran parte de los diccionarios aparecidos en la segunda mitad del XIX) no arrojan evidencias de un modelo claro.

En fin, desde el punto de vista de la historia de la lexicografía, el valor que puede tener esta obra se centra más en el conjunto del volumen (es decir en haber reunido tres parcelas diferentes) que en el vocabulario en sí, ya que los escolares disponían en el mercado de repertorios mucho más completos y útiles.

\section{Referencias bibliográficas}

Alvar Ezquerra, M. (1987): "Apuntes para la historia de las nomenclaturas en español". En Actas del VII Congreso Internacional de Lingüistica y Filología de América Latina (Alfal). Homenaje a Pedro Henriquez Ureña, Santo Domingo, Universidad Nacional Pedro Henríquez Ureña, I, págs. 457-470.

Ayala Castro, M. C. (1998): "Los otros diccionarios del español: clasificaciones metódicas del siglo XIX”, en M. Alvar Ezquerra y G. Corpas Pastor (coords.): Diccionarios, frases, palabras, Málaga, Servicio de Publicaciones de la Universidad, págs. 85-100.

Azorín Fernández, D. (1996): "La lexicografía española en el siglo XIX: del diccionario a la enciclopedia”, Estudios de Lingüística, 11, págs. 11-122.

Brunot, F. (1905-1979): Histoire de la lagnue française des origines à 1900. Tomo VIII, libro 2.2: "Le français en Espagne". París, Armand Colin.

Brunot, F. y Ch. Bruneau (1969): Précis de Grammaire Historique de la Langue Française, París, Masson et Cie.

Bruña Cuevas, Manuel (1996): "Le problème de l'orthographie française dans les grammaires à l'usage des Espagnols et dans les dictionnaires bilingües français-espagnol et espagnol-français (XVIe-XVIIIe siècles)". En E. Alonso, M. Bruña y M. Muñoz (eds.): La lingüística francesa: Gramática, hisioria y epistemología, I, págs. 85-101.

Bruña Cuevas, M. (2002): "Le [y] dans la pronociation figurée des dictionnaires français-espagnol des XIXe et XXe siècles”. En M. C. Figuerola M. Parra y P. Solà (eds.): La lingüística francesa en el nuevo milenio, Lleida, Milenio, Servicio de Publicaciones de la Universidad, págs. 123-133.

Bruña Cuevas, M. (2003): "Un diccionario bilingüe enciclopédico: Ramón Joaquín Domínguez, 184546)". En I. Iñarrea (et alii) (coords.): El texto como encrucijada: estudios franceses y francófonos, Logroño, Universidad de La Rioja, 2, págs. 283-294.

13 Carlos Soler Arqués, Novísimo diccionario manual franco-español e hispano-francés, Madrid, 1893. Sobre esta obra puede verse (Cazorla Vivas, 2005). 
Bruña Cuevas, M. (2005): "Histories des transcriptions phonétiques dans les dictionnaires françaisespagnol et espagnol-français”, Cahiers de lexicologie, 87-2, págs. 97-140.

Cabezas, M. I. y J. C. Herreras (1989): La enseñanza del francés en España, Valencia, Nau Llibres.

Cazorla Vivas, Ma . C. (2003): "Lexicografía bilingüe del siglo XIX: Ia primera edición del Diccionario francés-español y español-francés de R. J. Domínguez", en I. Doval y Ma R. Pérez (eds.): Adquisición, enseñanza y contraste de lenguas, bilingüismo y traducción, Vigo, Servicio de publicaciones d ela Unviersidad, págs. 63-70.

Cazorla Vivas, Ma. C. (2005): Lexicografia bilingüe con el español y el francés de los siglos XVIII y $X I X$, Madrid, Colección de Tesis Digital, Publicaciones Universidad Complutense.

Cazorla Vivas, Ma. C. (2006): "Diccionarios portátiles en el siglo XIX: la obra de C. M. Gattel (1798)". En Luque Durán, J. de D. (ed.): Actas del V Congreso Andaluz de Lingüística General. Homenaje al profesor José Andrés de Molina Redondo, III, Granada, Granada Lingvistica, págs. 1091-1102.

Esparza Torres, M. Á. (1999): "Notas sobre el Diccionario Nacional de Ramón Joaquín Domínguez": Estudios de Historiografia Lingüistica ofrecidos a Hans-Josef Niederehe, Vigo, Universidade de Vigo, págs. 39-63.

Fabbri, M. (1979): A Bibliography of Hispanic Dictionnaries. Catalan, Galician, Spanish, Spanish in Latin America and the Philippines, Ímola, Galeati.

Fernández Fraile, $\mathrm{M}^{\mathrm{a}}$ E. y J. Suso López (1999): La enseñanzalaprendizaje del francés como lengua extranjera en España (1767-1936). Estudio histórico: objetivos, contenidos y procedimientos, Granada, Método.

García Aranda, $\mathrm{M}^{\mathrm{a}}$. Á. (2005-2006): "La información fonética en la lexicografía menor del español: Le petit nécessaire des français qui vont en Espagne (1811)", Revista de Lexicografia, XII, págs. 159-176.

García Aranda, Ma . Á. (2006): "Una muestra de la lexicografía temática hispanoinglesa del siglo XIX". En Roldán, A. (coord.): Caminos actuales de la historiografia lingüistica: actas del $V$ Congreso Internacional de la Sociedad Española de Historiografía lingüistica, 1, págs. 623-636.

Lépinette, B. (1995): "La grammaire constrastive franco-espagnoles de la première moité du XVIIIe siècle", Analyse de six ouvrages édités en Espagne", Historiographia Lingüística, 22, 1/2, págs. 27.74.

Lépinette, B. (2000): "Gramáticas del francés editadas en España durante la primera mitad del siglo XIX”, Quaderns de Filologia. Estudis Lingüistics, V, págs. 177-195.

Palau y Dulcet, Antonio (1948-77): Manual del librero hispano-americano, Barcelona, Librería Palau.

Quilis, A. (1989): Bibliografia de la fonética y la fonología españolas, Madrid, CSIC.

Quilis Merín, M. (2004): "El modelo de la fraseología académica en el Diccionario Nacional (184647) de Ramón Joaquín Domínguez". En Corrales Zumbado (et alii): Nuevas aportaciones a la historiografia lingüistica. Actas del IV Congreso Internacional de la SEHL, II, Madrid, Arco/Libros, págs. 1345-1356.

Quilis Merín, M. (2007): “Las tareas lexicográficas en el siglo XIX: los diccionarios de Ramón Joaquín Domínguez". En M. Campos Souto, R. Cotelo García y J. Ignacio Pérez Pascual (eds.): Historia de la lexicografia española, Anexos de la Revista de Lexicografia, 7, La Coruña, Universidad, págs. 141-153.

Quemada, B. (1968): Les Dictionnaires du français moderne (1539-1863): Étude sur leer histoire, leurs types et leurs méthodes, París, Didier.

Seco, M. (2003): Estudios de lexicografía española, Madrid, Gredos.

Suárez Goméz, G. (1961): "Avec quels livres les Espagnols apprenaient le français (1520-1850)", Revue de Littérature comparée, 35, 1, págs. 158-171; 35, 2, págs. 330-346; 35, 3, págs. 512-523. 